\title{
The Research of the Emergency Rescue Model of the Freeway
}

\author{
Yi Zhang ${ }^{1, \text { a }}$, Yushu Zhang ${ }^{2}$ \\ ${ }^{1}$ Liaoning Provincial College Of Communications, Shenyang 110122, China \\ ${ }^{2}$ Tianjin University Of Technology And Education, Tianjin 300222, China \\ azhang e@126.com
}

\section{Keywords: Freeway; Accident; Emergency rescue; Dispatch; Model}

Abstract. There are so many people who are injured or dead in the traffic accidents due to not being rescued in time every year in China. Recently, death ratio per 10000 vehicles is 7.4 persons, and death ratio per 100,000 persons of traffic accident is 6.9 persons in China [1]. So the research of the emergency rescue model is necessary.This paper advances an emergency rescue model on the base of the Jilin province freeway, which provides the solution when accidents happen on the freeway. The model that minimizes the loss takes three factors into account, and they are the coverage gap defect, the loss per unit time and the travel time. There are also calculation methods of the three factors above all in detail. Finally, the paper gives the Hungary algorithm to solve the model.

\section{Review of Rescue Models}

In recent years, the research of the emergency rescue model has been going deeply.

Haghani advanced a dispatch and second time dispatch model at the 82nd annual meeting of the Transportation Research Board in Washington 2003. The model minimized the rescue time. It also took the changes of the traffic in account, so there was the second time dispatch in the model. For instance, the emergency vehicle 1 was sent to the accident $A$ and the emergency vehicle 2 was sent to the accident $\mathrm{B}$ to minimize the total rescue time. But the traffic conditions may change when the two rescue cars were traveling to the accidents, and the emergency vehicle 1 should be sent to the accident A and the emergency vehicle 2 should be sent to the accident B to minimize the total rescue time. So there would be the second time dispatch. This model minimized the total rescue time [2].

Saini Yang and Masoud Hamedi advanced a dispatch model which also minimized the total rescue time at 84nd annual meeting of the Transportation Research Board in Washington 2005. But in this model, rescue cars were classified into different types and a certain type vehicle was dispatched to a certain type accident. The model took the coverage gap defect into account, which meant the potential loss caused by the potential accidents due to the absence of the rescue car [3].

Minimizing the total rescue time in the emergency rescue model is not enough. In this paper, we would advance an emergency rescue model that minimizes the total loss of the accidents.

\section{Modeling}

The thinking of modeling. The modeling will be carried out in three cases in this paper. Firstly, let's look at the case 1 as the Fig. 1 . The accident $b_{1}$ happened between the rescue car $a_{1}$ and rescue car $a_{2}$, and $a_{1}$ was closer to $b_{1}$ than $a_{2}$ was. But $a_{2}$ was in charge of the section where the $b_{1}$ happened. Which rescue car should be sent? Obviously, $\mathrm{a}_{1}$ should be sent. So we think the travel time is important for the rescue strategy.

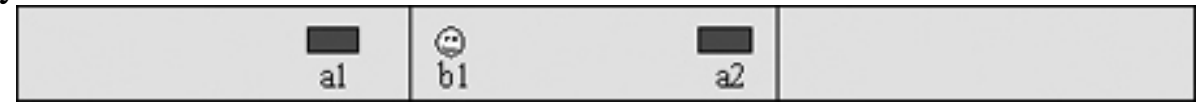

Fig. 1 An accident happened between two rescue cars (the coverage gap defect is not considered)

Secondly, let's look at the case 2 as the Fig. 2 . The case 2 is the same as the case 1 but the coverage gap defect is considered. According to the analysis above, the $a_{1}$ rescue car should be sent for the shorter travel time. While the $a_{1}$ was traveling to $b_{1}$, there would be another accident more serious 
happened in the section where the $\mathrm{a}_{1}$ vehicle was in charge of and the loss would be greater. We call the loss caused by the potential accidents due to the absence of the rescue car as the coverage gap defect. So, the coverage gap defect is important for the rescue strategy.

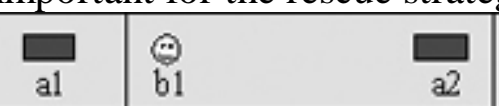

Fig. 2 An accident happened between two rescue cars (the coverage gap defect is considered)

Let's look at the case 3 as the Fig. 3. Accident $b_{1}$ happened between the rescue car $a_{1}$ and rescue car $a_{2}$, and the rescue car $a_{2}$ was closer to the accident $b_{1}$ than $a_{1}$. At the same time, another accident $b_{2}$ happened between the rescue car $a_{2}$ and the rescue car $a_{3}$, and the $a_{2}$ is closer to the accident $b_{2}$ than $a_{3}$. What was more important was that the accident $b_{2}$ was a very serious accident which would cause heavy loss if not handled. The $a_{2}$ rescue car was closer to the accident $b_{1}$ than to the accident $b_{2}$. So if we hope the total travel time to be shortest, we should send $a_{2}$ to $b_{1}$ and $a_{3}$ to $b_{2}$. But if the accident $b_{2}$ couldn't receive help in time the loss would be great. So we should send the rescue car $a_{2}$ to the accident $b_{2}$ and sent the rescue car $a_{1}$ to the accident $b_{1}$. So the severity is important for the rescue strategy. We use the loss per unit time $\mathrm{U}$ to represent the severity.

\begin{tabular}{|l|l|l|l|}
\hline al & b1 a2 & b2 & a3 \\
\hline
\end{tabular}

Fig. 3 Two accidents of different severity happened in three vehicles

From the analysis above, we can come to the conclusion that the rescue strategy is related to three factors. They are the coverage gap defect $\mathrm{D}$, the loss per unit time $\mathrm{U}$ and the total travel time $\mathrm{T}$. But what is the target of the rescue strategy? We think the rescue strategy should keep the total loss of the accident or accidents to a minimum. And the coverage gap defect $\mathrm{D}$, the loss per unit time $\mathrm{U}$ and the total travel time $\mathrm{T}$ are related to the loss in some way. That is to say, we can transform them to the loss as follows.

The calculation of the coverage gap defect $D$. This research is on the basis of the Jilin province freeway which is divided into ten parts, and there is one rescue car patrolling on each of these ten sections. We use A1 to A10 to represent the ten sections, and use the $\mathrm{a}_{1}$ to $\mathrm{a}_{10}$ to represent the ten rescue cars as the Fig. 4.

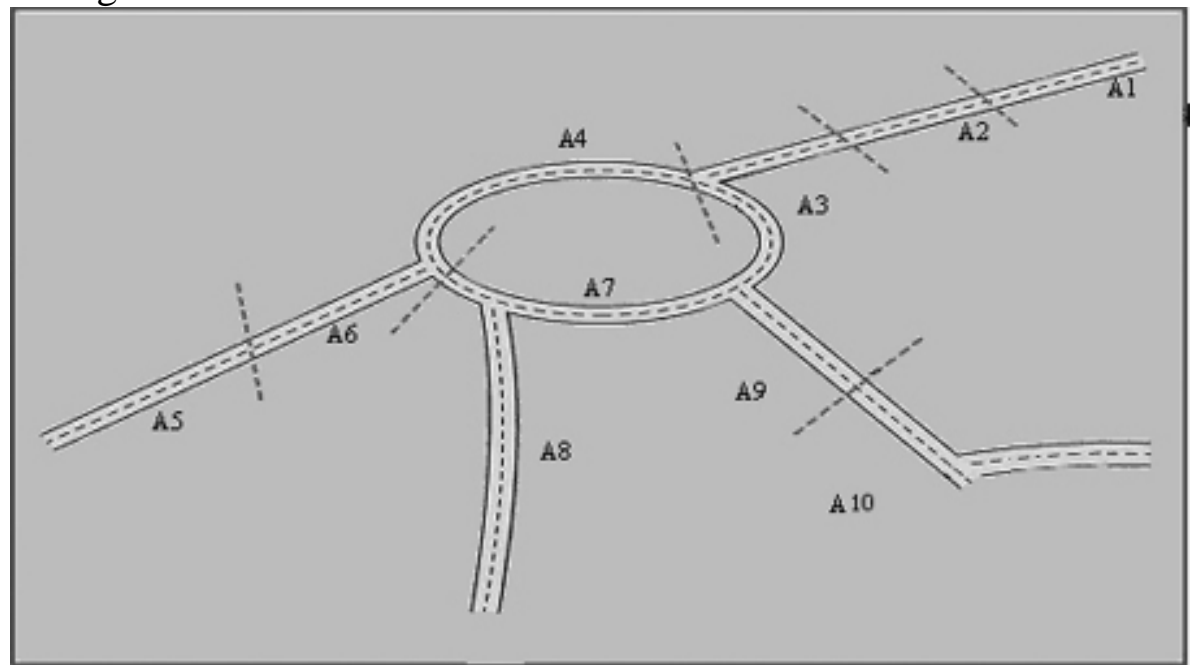

Fig. 4 Jilin freeway simple chart

To calculate the coverage gap defect $\mathrm{D}$, we collected the accident data of Jilin freeway for the last ten years from the Jilin freeway administrative department, and found that the amount and type of the accidents depended on the season. So we divided one year into four seasons, and calculate the average loss $\mathrm{L}$ of one day in a certain season. The loss $\mathrm{L}$ is the coverage gap defect $\mathrm{D}$ of a day in that season. If the rescue car is absent from its section for T hours, the coverage gap defect $\mathrm{D}$ would be $\frac{\mathrm{T} \times \mathrm{L}}{24}$. 
The calculation of the loss per unit time $\mathbf{U}$. We have collected the accident data for the last ten years from the Jilin freeway administrative department, and classified the accidents into different types. In addition, we classified a certain accident type into several severity levels and calculated the loss by the accident data. Every accident type of every level could indicate a loss L1. There is another parameter which we call as valuable time T1. If an accident happened and a person was injured who would survive if saved in six hours, the valuable time T1 is six hours. If the guardrail was destroyed, the valuable time T1 would be seven days or more. The valuable time is the useful rescue time of the accident. So the loss per unit time U would be L1/T1.

The calculation of the travel time $\mathbf{T}$. We could use the distance and speed to calculate the travel time. The simplest case is that the rescue car and the accident are on the same side of the road and the accident is in front of the rescue car. The distance divided by the speed equals the travel time. But it is not always so easy. If the rescue car is in front of the accident in the case above, the rescue car should cross the isolation strip twice and the distance would be more. If the rescue car and the accident are on the different side of the road, the rescue car should cross the isolation strip and the distance would be more too.

The formation of the model. Considering the coverage gap defect, the loss per unit time and the travel time, the loss of the rescue strategy would be $\sum_{n}(D+U T)$. The parameter $n$ is the amount of the accidents. The rescue strategy which minimizes the loss is what we are finding. Now, let's find the optimum rescue strategy from Table 1.

Table 1 The determinant of rescue

\begin{tabular}{|c|l|l|l|l|l|}
\hline Accident & $\mathrm{a}_{1}$ & $\mathrm{a}_{2}$ & $\mathrm{a}_{3}$ & $\mathrm{a}_{4} \ldots \mathrm{a}_{\mathrm{i}} \ldots \mathrm{a}_{9}$ & $\mathrm{a}_{10}$ \\
\hline $\mathrm{b}_{1}$ & $\mathrm{c}_{11}$ & $\mathrm{c}_{21}$ & $\mathrm{c}_{31}$ & $\mathrm{c}_{41} \ldots \mathrm{c}_{\mathrm{i} 1} \ldots \mathrm{c}_{91}$ & $\mathrm{c}_{10-1}$ \\
\hline $\mathrm{b}_{2}$ & $\mathrm{c}_{12}$ & $\mathrm{c}_{22}$ & $\mathrm{c}_{32}$ & $\mathrm{c}_{42} \ldots \mathrm{c}_{\mathrm{i} 2} \ldots \mathrm{c}_{92}$ & $\mathrm{c}_{10-2}$ \\
\hline $\mathrm{b}_{3}$ & $\mathrm{c}_{13}$ & $\mathrm{c}_{23}$ & $\mathrm{c}_{33}$ & $\mathrm{c}_{43} \ldots \mathrm{c}_{\mathrm{i} 3} \ldots \mathrm{c}_{93}$ & $\mathrm{c}_{10-3}$ \\
\hline $\mathrm{b}_{4}$ & $\mathrm{c}_{14}$ & $\mathrm{c}_{24}$ & $\mathrm{c}_{34}$ & $\mathrm{c}_{44} \ldots \mathrm{c}_{\mathrm{i} 4} \ldots \mathrm{c}_{94}$ & $\mathrm{c}_{10-4}$ \\
$\ldots$ & $\ldots$ & $\ldots$ & $\ldots$ & $\ldots \ldots \ldots \ldots \ldots$ & $\ldots$ \\
$\mathrm{b}_{\mathrm{j}}$ & $\mathrm{c}_{1 \mathrm{j}}$ & $\mathrm{c}_{2 \mathrm{j}}$ & $\mathrm{c}_{3 \mathrm{j}}$ & $\mathrm{c}_{4 \mathrm{j}} \ldots \mathrm{c}_{\mathrm{ij}} \ldots \mathrm{c}_{9 \mathrm{j}}$ & $\mathrm{c}_{10-\mathrm{j}}$ \\
$\ldots$ & $\ldots$ & $\ldots$ & $\ldots$ & $\ldots \ldots \ldots \ldots \ldots$ & $\ldots$ \\
$\mathrm{b}_{\mathrm{n}}$ & $\mathrm{c}_{1 \mathrm{n}}$ & $\mathrm{c}_{2 \mathrm{n}}$ & $\mathrm{c}_{3 \mathrm{n}}$ & $\mathrm{c}_{4 \mathrm{n}} \ldots \mathrm{c}_{\mathrm{in}} \ldots \mathrm{c}_{9 \mathrm{n}}$ & $\mathrm{c}_{10-\mathrm{n}}$ \\
\hline
\end{tabular}

The $a_{i}$ is the rescue car; the $b_{j}$ is the accident; the $c_{i j}$ is the loss when $a_{i}$ is sent to handle $b_{j}$; and the $\mathrm{n}$ is the amount of the accidents. We just want to find $n \mathrm{c}_{\mathrm{ij}} \mathrm{s}$ which are in different rows and columns to minimize the $\sum \mathrm{c}_{\mathrm{ij}}$. The rescue is that the $\mathrm{a}_{\mathrm{i}}$ is sent to handle $\mathrm{b}_{\mathrm{j}}$. The problem can be transformed into mathematics model:

$$
\min \sum_{\mathrm{i}=1}^{10} \sum_{\mathrm{j}=1}^{\mathrm{n}} \mathrm{k}_{\mathrm{ij}} \mathrm{c}_{\mathrm{ij}}
$$

where $\mathrm{k}_{\mathrm{ij}}=1$ if $\mathrm{a}_{\mathrm{i}}$ is sent to $\mathrm{b}_{\mathrm{j}}$

$\mathrm{k}_{\mathrm{ij}}=1$ if $\mathrm{a}_{\mathrm{i}}$ is not sent to $\mathrm{b}_{\mathrm{j}} \quad(\mathrm{i}=1, \ldots, 10 ; \mathrm{j}=1, \ldots, \mathrm{n})$

$\sum_{\mathrm{i}=1}^{10} \mathrm{k}_{\mathrm{ij}}=1(\mathrm{i}=1, \ldots, 10) \quad \sum_{\mathrm{k}=1}^{\mathrm{n}} \mathrm{k}_{\mathrm{ij}}=1(\mathrm{j}=1, \ldots, \mathrm{n})$ 
To solve the model, we could use the Hungary algorithm of Operation Research [4] and computer program.

\section{Conclusions}

This research advanced a rescue model which minimized the loss when the several accidents happened at the same time on the freeway. The model analyzed the coverage gap defect, the loss per unit time and the travel time. But it ignored another case that the accidents happened at different time, and that was what we would research next.

\section{References}

[1] Information on http://www.moc.gov.cn

[2] Haghani, A., H.Hu, and Q.Tian: the $82^{\text {nd }}$ Annual Meeting of the Transportation Research Board(Washington,D.C.,2003).

[3] Saini Yang,Masoud Hamedi and Ali Haghani: the $84^{\text {th }}$ Annual Meeting of the Transportation Research Board(Washington,D.C., 2005).

[4] Yunquan hu: Operation Research(Harbin Institute of Technology Press, China 1985). 